\title{
ESPACIO ARQUITECTONICO: OBJETO DE COMUNICACION Y EXPERIENCIAS INTANGIBLES ${ }^{1}$
}

\author{
Recibido: 20 de Agosto de 2015 - Aceptado: 20 de Noviembre de 2015 - Artículo de Reflexión
}

\author{
Mauricio Cabas García \\ Universidad de la Costa-CUC. Barranquilla, Colombia.mcabas1@cuc.edu.co
}

Para citar este artículo / to reference this article:

Cabas, M. (2016). Espacio arquitectonico: objeto de comunicacion y experiencias intangibles. Módulo Arquitectura CUC, Vol.16 N¹. 135-152.

\section{Resumen}

Este artículo tiene como objetivo presentar una reflexión sobre la evolución del concepto de espacio arquitectónico, a través de la historia, desde una perspectiva fenomenológica y como objeto de comunicación. La conceptualización del espacio arquitectónico no ha sido única a través del tiempo, por el contrario ha sido un proceso de cambios ideológicos que dependen generalmente de la variedad de culturas y de pensamiento. En estos casos la percepción como experiencia sensorial tiene un rol protagonista pero no se puede dejar a un lado el fenómeno de la experiencia espacial, que consiste en todas las vivencias, recuerdos, memorias, sentimientos que se asociación a un lugar o espacio. Esto nos lleva al tema de la fenomenología del espacio arquitectónico, a la idea de la poética del espacio de Gastón Bachelard ${ }^{3}$ y al concepto de atmosfera de Peter Zumthor. ${ }^{4}$

\section{Palabras clave}

Espacio arquitectónico, fenomenología, experiencia espacial, concepto, objeto.

\footnotetext{
1 Articulo producto de la investigación terminada en 2009 llamada "Criterios de diseño arquitectónico de espacios que intensifiquen la conducta creativa" financiada por la Corporación Universidad de la Costa- CUC, Barranquilla- Colombia

2 Arquitecto y especialista en Diseño Arquitectónico de la Universidad Autónoma del Caribe. Candidato a magister en proyectos de Arquitectura y Urbanismo de Funiber junto con la Universidad Unini de Puerto Rico. Docente de tiempo completo de la Universidad de la Costa (CUC), Barranquilla, desde el año 2007. Cofundador de la empresa Cabas/Garzon Arquitectos en 2010. Correo: mcabas1@cuc.edu.co, mauricio@cabasgarzon.com

3 Gaston Bachelard Gaston Bachelard (1884- 1962) filósofo, poeta, físico, profesor y crítico literario. Siempre estuvo interesado por la historia de la ciencia, moderna o contemporánea, y al mismo tiempo por la imaginación literaria. Su obra "La poética del espacio" expone un concepto metafísico del espacio; proponiendo ideas bastante minuciosas acerca de todos sus rasgos y jerarquías internas.

4 El arquitecto suizo Peter Zumthor en su conferencia titulada "Atmosferas. Entornos arquitectónicos. Las cosas a mi alrededor" expresa que el concepto de atmosfera se refiere a una sensibilidad emocional que sentimos al estar en contacto con el espacio arquitectónico existente que tiene que ver no solo con la percepción sino con otros distintos factores; algo de magia, algo de misterio y una armonía comparables con las composiciones de la música clásica.
} 


\title{
ARCHITECTURAL SPACE: OBJECT OF COMMUNICATION AND INTANGIBLE EXPERIENCES
}

\begin{abstract}
This article aims to present a reflection on the evolution of the concept of architectural space, through history, from a phenomenological perspective and as an object of communication. The conceptualization of the architectural space has not been unique over time, on the contrary it has been a process of ideological changes that generally depend on the variety of cultures and thought. In these cases, perception as a sensory experience has a protagonist role, but we can not leave aside the phenomenon of spatial experience, which consists of all the experiences, memories, memories, feelings that are associated with a place or space. This brings us to the subject of the phenomenology of the architectural space, to the idea of the poetics of the space of Gastón Bachelard and to the concept of atmosphere of Peter Zumthor.
\end{abstract}

\section{Keywords}

Architectural space, phenomenology, spatial experience, concept, object. 


\section{Introducción}

Cada vez que nos enfrentamos a un espacio arquitectónico, la experimentación del mismo nos lleva a un estado psicofísico que puede describirse como místico. Aunque generalmente estos eventos se mantienen ocultos, debido a que incurrimos en la idea de subestimarlos y temiendo a ser ridiculizados, además, que en el ámbito profesional y académico estos conceptos no están aceptados. Muchos arquitectos reconocidos han dado manifiesto de estas experiencias intangibles a pesar de los riesgos a los que se podrían haber enfrentado, uno de estos testimonios fue realizado por Le Corbusier (Le Corbusier, 1993) Lo importante de esos testimonios es su consistencia al describir situaciones que desafían lo que se conoce como arquitectura y dejan claro las sensaciones de la disolución de la división entre sujeto-objeto, sensaciones de bienestar, intuiciones profundas sobre la vida, efectos anormales de espaciotiempo, amor completo por todo lo que existe y la ineficiencia del lenguaje para expresar lo experimentado (Bermúdez, 2014). De igual manera, el hecho arquitectónico solo podría considerarse completo al momento que el ser humano interviene en él y lo experimenta, solo cobra relevancia y vida propia en correspondencia con la percepción del ser humano que lo percibe. (Ando, 1995). No basta solo con ver el espacio arquitectónico sino que este debe experimentarse, tratando de apreciar cómo fue concebido, cuál fue su propósito y la correspondencia al estilo y a la época. Hay que habitar los espacios de cada obra arquitectónica, percibir como nos envuelven, como nos van guiando sin darnos cuenta (Rasmussen, 1980). En los últimos años han salido al escrutinio público nuevas investigaciones que dan soporte empírico a estos testimonios. (Bermudez, 2008) Muy a pesar de que siempre se trata de categorizar las experiencias espaciales como algo que hace parte de lo estético, en realidad esas experiencias desafían la definición de estética en las corrientes filosóficas y culturas modernas. La definición de lo estético solo se limita a una percepción del significado de la belleza que fue concebido en el siglo XVIII. (Gadamer, 1968) Pero en realidad es todo lo contrario, la experiencia espacial arquitectónica no está limitada solo a la percepción, sino que está ligada a sentidos de realidad inseparable de lo bello, lo verdadero, lo bueno y lo místico y en realidad está estrechamente influenciada por la posición física y tridimensional del ser que habita y aprecia el espacio. 


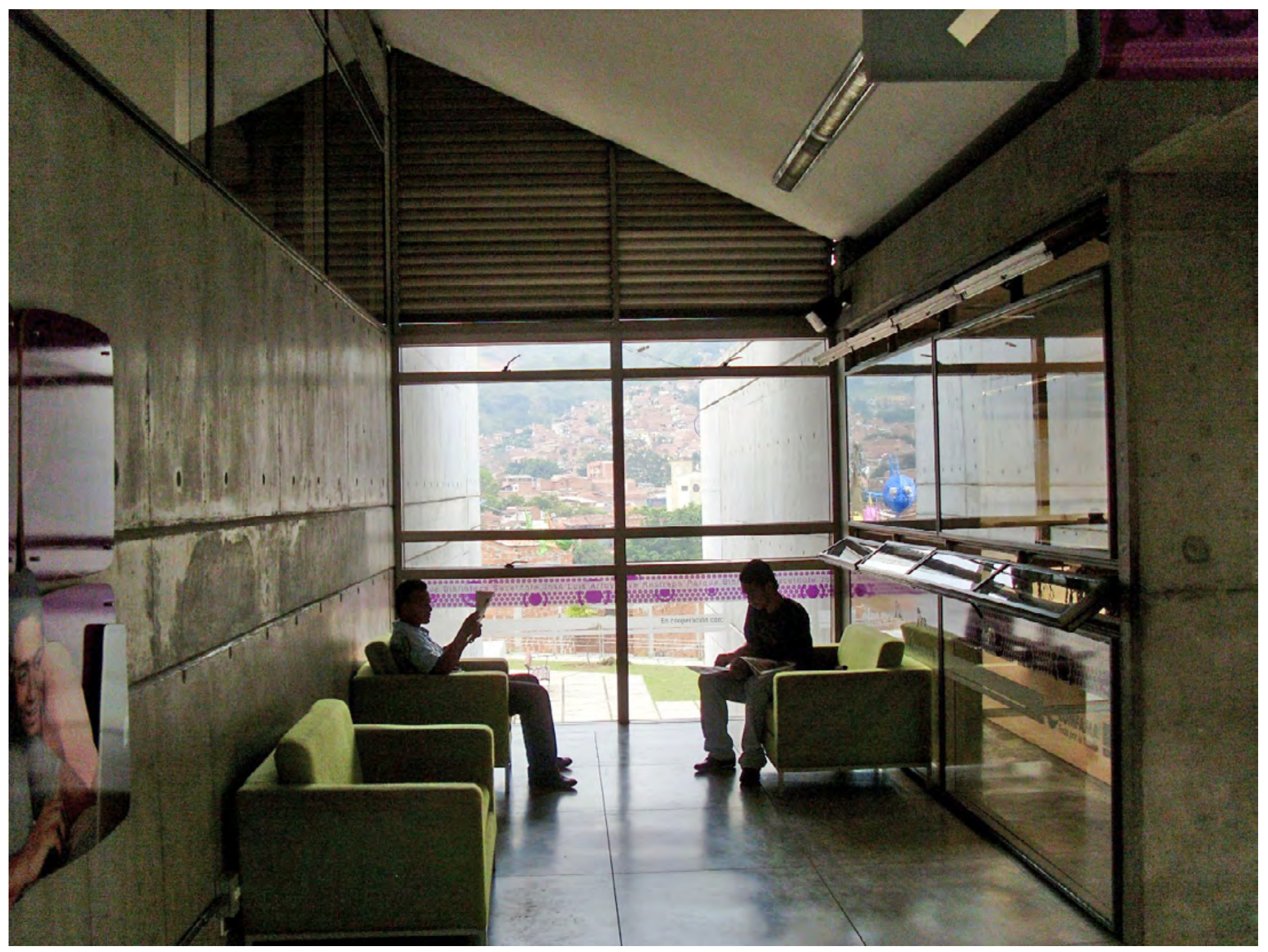

Figura 1. Biblioteca San Javier, Medellín 2009.

El juego de luz y sombra generado por la ventana retraída genera reflejos en el piso y una sensación de tranquilidad 
(Bermúdez, 2014). El concepto de habitar, radica en movernos dentro del espacio de una construcción, caminar, recorrer, analizar, entre otras cosas, obteniendo múltiples puntos de vistas, distintas perspectivas de un mismo objeto y la experiencia del paso del tiempo. Aunque no debemos confundir estas experiencias espaciales con ideales platónicos, lo experimentado al momento de situarnos en un espacio va directamente relacionado a una visión Zen ${ }^{5}$ de la realidad. Esto implica el sumergirse en lo que se está viviendo, sentir, palpar, tanto así que nos volvemos uno con el espacio, la relación sujeto y objeto se intensifica, desplegando solo así una experiencia consciente (Bermudez, 2014).

Desde el momento que hablamos sobre espacio, no estamos hablando sobre una realidad definida ni objetiva, por lo contrario estamos hablando de un concepto que se ha venido desarrollando desde hace muchos años y sus manifestaciones se aprecian en los cambios de las formas arquitectónicas en la historia. Podemos considerar que el espacio es un concepto, sabemos que existe en una dimensión

5 Es una escuela del pensamiento budista, la cual se aparta del conocimiento teórico e intelectual, y por el contrario busca la experiencia de la sabiduría más allá de lo racional. física, pero su entendimiento puede ser interpretado de distintas maneras, desde puntos de vista filosóficos hasta geométricos, pasando por conceptos fenomenológicos hasta meramente funcionales y depende de le época histórica de su contexto. Esta conceptualización del espacio tiene varios componentes, uno de los más importantes es el concepto del espacio como representación de la naturaleza como explica Giulio Carlo Argán. (Argan, 1984) A principios de los años 600, la arquitectura es pensada como representación del espacio, es decir, el arquitecto de la época considera que está representando con su edificio una realidad. El concepto va evolucionando en el tiempo hasta pensar que en la realidad se va creando el espacio a partir de la ejecución de la arquitectura. (Argan, 1984) Entre estas dos consideraciones del concepto del espacio existen grandes diferencias, empezando con el método que utiliza el arquitecto. El arquitecto que representa el espacio está sujeto a utilizar elementos formales predeterminados y que están a su disposición, componiendo su edificio basado en la combinación de los elementos y formas arquitectónicas preestablecidas. De igual manera toma interpretaciones de las formas de la naturaleza y conceptos de la historia. 


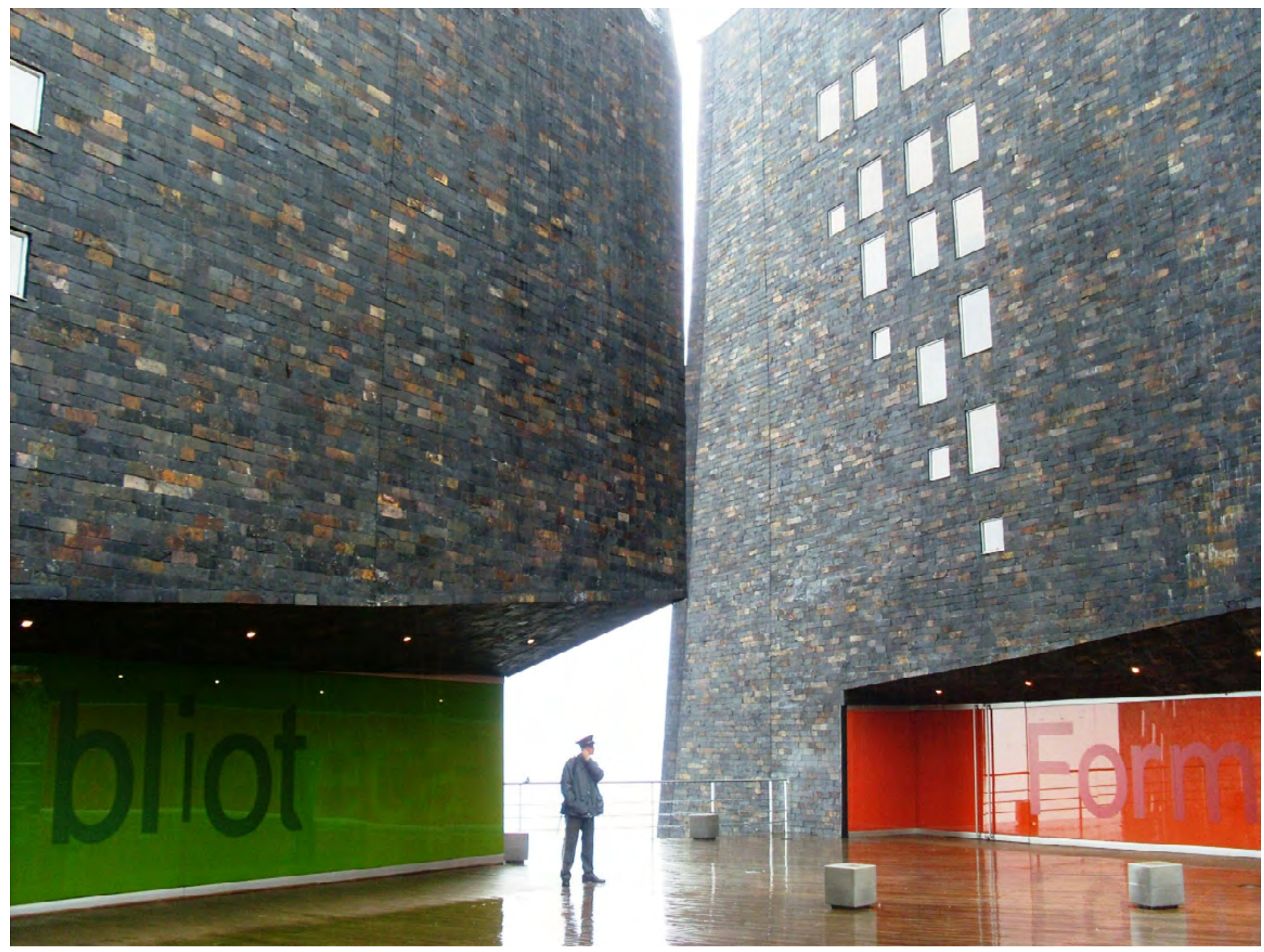

Figura 2. Parque Biblioteca España, Medellín 2009 La composición volumétrica junto con la entrada de luz, los colores oscuros de las piedras pizarras y la ventaneria de color, dan la sensación de proporciones enormes que se 
Esto no significa que sea una arquitectura de repetición, recordemos que cada interpretación cambia según el individuo y la época. Por otra parte el arquitecto que pretende hacer el espacio, no acepta formas predeterminadas, sino que tendrá que crear sus formas arquitectónicas, esto se conoce como arquitectura de determinación formal espacial, no recurre a ninguna premisa histórica u objetiva, y el espacio se crea con la evolución de una idea de la forma arquitectónica propia. Existen tres etapas de conceptualización del espacio arquitectónico; el primero que empieza con los imperios antiguos hasta el imperio griego. La segunda etapa comprende desde el imperio romano hasta mediados del siglo XX, y la tercera etapa se identifica con las conceptualizaciones modernas de arquitectos como Le Corbusier, Frank Lloyd Wright y Mies Van der Rohe. (Giedion, 1969)

En la antigüedad, los asentamientos eran construidos dentro de murallas muy fortificadas, trayendo como resultado, espacios estrechos, la arquitectura más que buscar una buena concepción del espacio, se enfocaba en la construcción de Templos y Palacios para Dioses y Reyes respectivamente. (Cabas, 2010). En la arquitectura egipcia, el concepto del espacio interior solo tenía sentido si permitía el desplazamiento o movimiento dentro del edificio hasta llegar a un punto importante del mismo (Moline, 2011).

Las culturas prehispánicas en América manejaban un concepto de espacialidad urbana de grandes proporciones muy organizada, con grandes plazas ceremoniales y centros de reunión de población. Un ejemplo de esto se dio en Teotihuacán, en donde se encontró un concepto básico de planificación con una composición espacial según medidas supuestamente divinas. (Munizaga, 1997) Lo que importaba en estos lugares era el conjunto en el exterior. Los nativos de América precolombina siempre vivían en armonía con la naturaleza y poseían una excelente relación con su entorno, lo respetaban, pero solo representaban una realidad fuera de sí mismos mas no hacían el espacio (Cabas, 2010) imponiendo criterios preconcebidos para llegar a una claridad netamente funcional, con algunos efectos estéticos y circulaciones racionales. (Hardoy, 1968)

En Europa, el templo griego ignoraba por completo el espacio interior y por el contrario exaltaba la escala humana. Esta arquitectura era escul- 


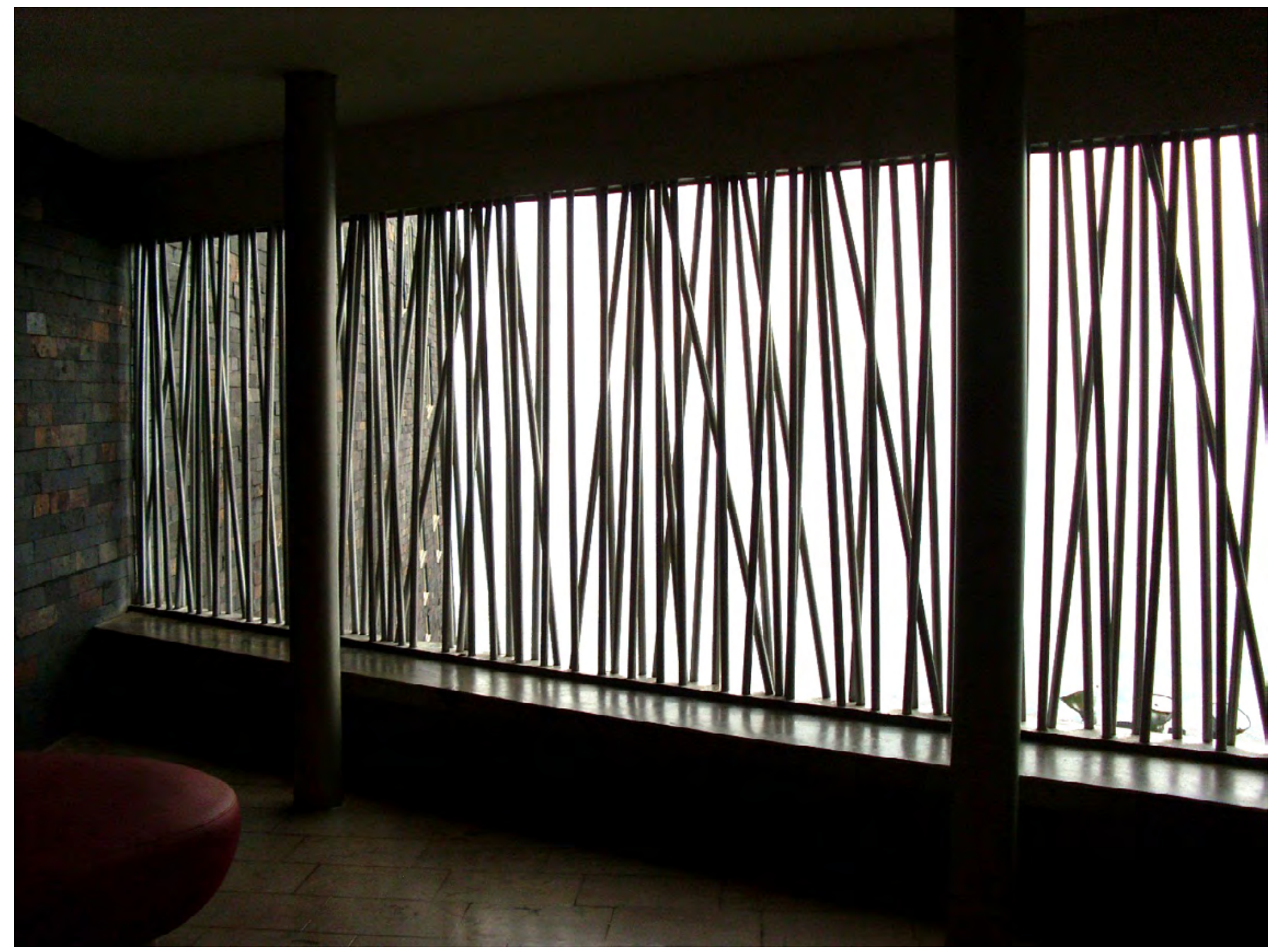

Figura 3. Parque Biblioteca España, Medellín 2009.

Los elementos verticales permiten la entrada de luz de forma tamizada, dejando el espacio casi en penumbras, dándole una sensación de misterio y misticidad al espacio interior. 
tural, se concibió como escultura, para ser disfrutada desde la lejanía valorando la proporción, la escala y su compenetración con el entorno, no existía concepción del espacio arquitectónico interior, era en realidad un espacio residual cerrado sin ninguna relación con el exterior. Este espacio interior nunca fue concebido ni pensado, porque no respondía a funciones sociales. (Zevi, 1958)

La vida social griega se generó toda en el exterior, al aire libre rodeada de un entorno impresionante, no hay que olvidar donde departía con sus discípulos en gran Sócrates, a la sombra de un Olivo. Tal vez los recorridos marcados por los cambios axiales tuvieron algo que ver con los progresos intelectuales, filosóficos y creativos de la época. Y es que "los griegos querían ser bañados por la luz". (Barreneche, 1996) El espacio interior era oscuro, sin ningún concepto compositivo y sin ningún valor socializador, el hombre solo lo rodea pero nunca lo disfruta, y se entendía como el vacío que existía entre los volúmenes (Cabas, 2010).

En Roma, el espacio arquitectónico si fue pensado, aunque era un espacio interior estático, gobernado por la simetría y la escala monumental, generando la sensación de majestuosidad, de superioridad y autoridad. Pero al contrario del espacio griego, en el espacio interior romano generalmente de planta circular y rectangular si se podía deambular. Era un espacio unitario y direccionado muy posiblemente concebido para subyugar intencionalmente, debido al esquema de jerarquización que existía. El habitante del imperio romano vuelve al espacio interior para reencontrarse consigo mismo, con su familia y sus antepasados y de ese modo lograr armonía en su vida. En este punto empieza la conquista verdadera del espacio interior, en cual se puede moldear según la intención de quien lo crea. Con la construcción de la bóveda y la cúpula el espacio interior ganó relevancia, convirtiéndose no solo en un espacio con dirección horizontal, sino que aparece la dirección vertical. El ingreso de la luz permite la sensación de reanimación del espacio ofreciéndole color, poco a poco este espacio interior romano se va iluminando, tratando de imponer los criterios políticos e institucionales del imperio como la claridad de actuación, de sabiduría y de poder. Es un espacio lleno de significados, generalmente era un espacio sociopeta, concebido para estar y no para recorrer. (Cabas, 2010) 
Con el inicio del cristianismo, nacieron espacios de reunión, buscando un espacio físico más humano para encontrarse y refugiarse de la persecución del imperio romano. Se trató de compenetrar conceptos griegos en términos de escala y en términos de espacio interior parecido al romano. En ese instante ya se trataba de moldear de alguna manera el comportamiento de los creyentes dentro del templo, se nota la direccionalidad horizontal del espacio cristiano enmarcado con líneas horizontales que acompañan el camino del hombre, precisamente lo que quería demostrar la nueva doctrina cristiana, luego de años y años de persecución. En este periodo se produce un hecho fundamental en la conceptualización del espacio arquitectónico, la arquitectura ya no es pensada solo como representación del espacio sino como determinación del espacio mediante las formas arquitectónicas. (Cabas, 2010)

En la época del imperio bizantino, la preocupación principal era la dinámica dentro del espacio, dejando atrás la estaticidad del espacio interior, generando ritmo, permitiendo una aceleración en los recorridos, repitiendo arcos, ventanas y otros buscando la constante dilatación del mismo, ampliarlo desde su centro. El espacio bizantino nace de una inspiración nueva, de una espiritualidad abstracta. En la cultura bizantina se mezcla el concepto físico y metafísico y el espacio arquitectónico busca representar esa mezcla de una forma coherente. (Cabas, 2010)

En el espacio Carolingio se presenta un cambio radical manejándose dos tipologías, planta rectangular donde el pueblo ora y se realizan las ceremonias religiosas y planta circular, para los espacios donde reside el emperador. Estas tipologías demuestran una clara referencia en la generación de sentimientos en la persona que los experimentaba. La primera era el acompañamiento del ser y la segunda en que la habita el emperador representa la eternidad. Ya a mediados del siglo XI se llega a la maduración del espacio románico, en el cual la dimensión métrica toma mucha importancia en la concepción del mismo. El espacio románico es medido completamente, no es arbitrario ni dejado a la suerte, manifestándose tridimensionalmente, es más complejo, tratando de generar sensaciones y actitudes psicológicas, creando claros y oscuros, todo esto gracias el desarrollo tecnológico y avances constructivos. (Cabas, 2010) 
En el gótico todo se vuelve más subjetivo. El espacio se convierte en inconmensurable, infinito y disperso, el sueño de los arquitectos góticos era crear un espacio rítmico, elevarlo y darle forma sin interrumpir su continuidad, contrario a la escala humana generando en la persona que lo experimenta un estado de ánimo desequilibrado. Es un espacio vivo construido para trasmitir sentimientos de trascendencia, donde la subjetividad e inmaterialidad eran predominantes. (Cabas, 2010)

Se pretendió utilizar la menor estructura posible de los muros de cerramiento con el fin de lograr una identificación del espacio religioso interior por medio de la luz, la cual se manejaría tamizada para acentuar la idea de trascendencia. La arquitectura gótica está empeñada en romper la gravedad, pretendiendo significar el milagro de que un espacio está por encima de lo material. En cierto sentido no interesa el conocimiento técnico sino las leyes divinas y absolutas, de ahí que el concepto del arquitecto medieval no era ser original ni pretendía crear formas nuevas. El arquitecto no tenía la obligación de crear sino de formalizar la idea o el mito de la religión. (Goycoolea, 1998)
En la grandes catedrales góticas se logró, gracias a la esbeltez, a su luminosidad, y con una especial al cromatismo de los vitrales, una refinada atmósfera espiritualizada que buscaba como fin la belleza inconmensurable, a pesar de la materialidad en piedra se convierte en una estructura diáfana, transparente, que representa toda la desmaterialización de la teología cristiana medieval. Se conformaba un escenario que despertaba en el habitante un sentimiento de sobrenaturalidad y trascendencia; pretendiendo llevar a que el hombre medieval elevará su mirada hacia un vacío grandioso y luminoso, y sintiera que iba ascendiendo humildemente hacia el cielo. (Grillo, 2005)

Tras un largo periodo medieval, todos los grandes arquitectos volvieron sus miradas a Roma, lo que permitió retomar todos los conceptos arquitectónicos y estéticos que allí se habían originado. Estos arquitectos no solo tomaron estos conceptos sino que los interpretaron para generar su propio estilo de vida (Cabas, 2010). El Espacio del Renacimiento nacía el cual es producto de la innovación desde el punto de vista espiritual y psicológico. Es un espacio para el hombre, para que el hombre lo domine. (Zevi, 1958) No busca un éxtasis religioso, sino en 
un régimen métrico tanto en planta como en volumetría, la concepción del espacio es abstracta, se basa en la utilización de la proporción Áurea, con el fin de acercar al hombre al conocimiento, al pensamiento, al control intelectual del mismo sobre todo lo demás. El significado del espacio es el de construir un nuevo hombre a través de la libertad, generándole un microcosmos donde se sienta a gusto reduciendo todo a los conceptos matemáticos y armónicos del universo. Existe el principio de unidad y de un mundo sensible, la arquitectura se ve como una ciencia matemática para hacer visible el orden cósmico. Se genera una iluminación racional, clara y transparente, se crea un espacio sin contraste de luz, todo se puede ver claramente (Cabas, 2010). La luz se convierte en instrumento para poder comprender la realidad. (Barreneche, 1996).

En el siglo XVI se visualiza un espacio absoluto, fácil de captar desde cualquier punto caracterizado por equilibrios de proporción. Es un espacio simétrico con esquema central y circular, espacialmente no hay mayor movimiento pero si un sentido de pesadez, convirtiéndose en un objeto escultórico y a la vez dramatismo, dado por las pinturas en sus paredes y techos, lleno de luces y sombras. Cuando el espacio barroco incurre, llega con la necesidad de romper, abrir, alejar, derribar, con un grito de rebelión, llevando consigo un significado psicológico, induciendo a sentimientos de libertad y movimiento. Se concibió para transmitir al que lo experimenta, sentimientos de conmoción, dramatismo, realismo y trascendencia con el objetivo de que el hombre tratará de comprender su mundo, y al mismo tiempo comprender a Dios a través de los sentidos y la razón (Cabas, 2010).

En el siglo XIX el concepto de espacio no tuvo ningún desarrollo, lo que se logra es un espacio mediocre con esterilidad poética. Todo lo contrario al concepto de espacio en la era moderna, el cual se fundamenta en la planta libre, se vuelve a pensar en el sentido espacial, explotando los avances tecnológicos y logrando gran integración entre el interior y el exterior, provocando grandes ventanales, paredes interiores delgadas y movibles, permitiendo la continuidad espacial, tratando de generar la mayor amplitud posible. En esta época la conceptualización como objeto de comunicación del espacio se ve influenciada en gran manera 
por los artistas plásticos, lo que trae la introducción de conceptos como espacio continuo, paisaje subrayado, transparencia total, descomposición de la caja, plano flotante y espacio adintelado (Cabas, 2010). En esta nueva visión de la realidad, y describiendo la arquitectura como un objeto fenomenológico el sentido de la gravedad pierda valor. Y empieza a delinearse una nueva estática de las horizontales que, tal como defenderá la Bauhaus, busca contrarrestar la gravedad misma. La simetría bilateral se pierde. El espacio se expresará mediante un equilibrio asimétrico y rítmico, multiplicidad de ejes, distintos centros de equilibrio, superando la inercia y la forzada composición de su predecesor. Visto como fenómeno el espacio, superará todos los discursos programáticos, manifiestos y polémicas sobre la arquitectura moderna. La nueva idea de estática fundamentada en la Lebenswelt ${ }^{6}$ va más allá del concepto de gravedad tradicionalmente expresada en las verticales, reconociendo otras fuerzas, manifiestas en la tensión interna y recíproca de las masas, la unidad y la continuidad de la propia realidad Esto trae como consecuencia una

6 El concepto expuesto Edmund Husserl refiriéndose a todos los actos culturales, sociales e individuales de los cuales nuestra existencia no puede sobrepasar modificación en la conciencia de que nuestro ser ya no precisa de antítesis del no-ser, permitiendo que un problema ontológico se exprese en la arquitectura contemporánea a través de una formulación fenomenológica. (Alvarez, 2013) Es un espacio medido cuidadosamente, logra gran simplicidad y al mismo tiempo dramatismo. Esto impulso a que Marcel Breuer ${ }^{7}$ indagará sobre las líneas tensas y las curvas elásticas que acompañan los movimientos espontáneos del cuerpo humano en un encuentro de coordenadas, un abstracto lugar espacial al que la propia subjetividad dará vida y concreción. Lo que pone en entre dicho la conjetura del ser en el espacio como un simple encuentro de coordenadas ya que la posibilidad de replegarse, desaparecer y volver a entrar en el vacío, bajo la creencia de que los objetos y todo lo que los rodea solamente existen cuando nos aproximamos, los usamos o manipulamos, empezando y terminando con una serie de actos de nuestra propia vida en los que intervienen las ideas de nuestra propia subjetividad. (Alvarez, 2013).

7 Marcel Breuer (1902 -1981) arquitecto y diseñador industrial considerado como uno de los principales maestros del modernismo y que además se interesó en gran medida por la construcción modular y las formas simples. 
Del espacio orgánico, su máximo exponente fue Wright, creando un espacio lleno de movimiento, de direccionamientos, tratando de generar ilusiones con distintas perspectivas, su objetivo no era impresionar el ojo del hombre, sino expresar la acción misma de su vida. La ondulación ya no se expresa solamente como una visión artística de comunicar movimiento sino para hacer un acompañamiento en el camino del ser humano. (Zevi, 1958).

Por otra parte, Le Corbusier, considerado como el arquitecto más importante del modernismo, se fundamenta en el manejo de elementos naturales como el sol, luz, vegetación y libertad plena del espacio (Cabas, 2010). Su arquitectura se define dentro de los perfiles expresionistas y será la luz el elemento que defina la presencia y fuerza al espacio interior. El espacio se vuelve el protagonista principal de la arquitectura, se interpreta como un hecho concreto que debe generar emoción y sentimientos, y con el manejo de la luz natural se logra esto. En 1954 cuando publica The Modulor, declaró: "La naturaleza es ley y orden, unidad e interminable diversidad, sutileza, armonía y fuerza" (Le Corbusier, The Modulor, 2000). La luz se convierte en un material arquitectónico.

A partir de ese momento el concepto de espacio es pensado en términos fenomenológicos y surge una minoría de arquitectos que buscaban hacer converger y relacionar el racionalismo con algo de expresionismo, con algo de arte informal y constituida en la idea de deconstruir (Zevi, 1999). Uno de estos arquitectos es Frank Gehry, haciendo de la intuición la clave imprescindible de la creación del espacio arquitectónico, fundamentado en asimetrías y disonancias, y pensado para ser admirado desde distintos puntos, siempre siendo diferente buscando el asombro y protegido por una envoltura espontanea, y al mismo tiempo siendo completamente funcional (Cabas, 2010). Una de las características importantes del trabajo de Frank Gehry es la majestuosa espacialidad interior, la tridimensionalidad y el sentido del lugar. Sus propuestas nacen de una experimentación volumétrica que va conformando un espacio interior que se puede envolver prácticamente con lo que sea, cualquier revestimiento que permita comunicar algo (Garzon, 2012).

La arquitectura contemporánea se enmarcará en los conceptos fenomenológicos, generando una profunda transformación, con la cual, el edificio adquiere un valor de determinación espacial como un valor estético, sólo para quien habite su espacio sin poder objetivarlo, como en el resto de las obras de arte. Aquí podemos identificar los conceptos kantianos de la 
tercera Crítica, en la cual se expresa este proceso fundamentado en hecho del juzgar reflexionante, asociando el placer estético con el intento de asimilar las relaciones lógicas de las que surge la forma de un objeto. El placer estético se concreta como una experiencia consciente, un juego libre de entendimiento e imaginación en el que no importan los objetos en sí, o como interpretaba Kant como una "vivificación de las facultades cognoscitivas"8. (Alvarez, 2013)

\section{Conclusion}

La fenomenología, en cierta manera representa la intuición expresada como una actitud propia del pensamiento al enfrentarse a ciertos fenómenos, determinada por una condición de la conciencia, una visión primordial del cómo o el modo en que las cosas u objetos o construcciones se revelan ante nosotros. La esencia de la arquitectura como en la esencia de todas las artes, es el resultado intencional de los actos subjetivos que dan sentido a las construcciones, revelándose estas como productos de un proceso de creación. (Alvarez, 2013)

8 Se refiere Kant al entendimiento e imaginación, producido por el placer estético relativo al bello, que puede alcanzar lo sublime.

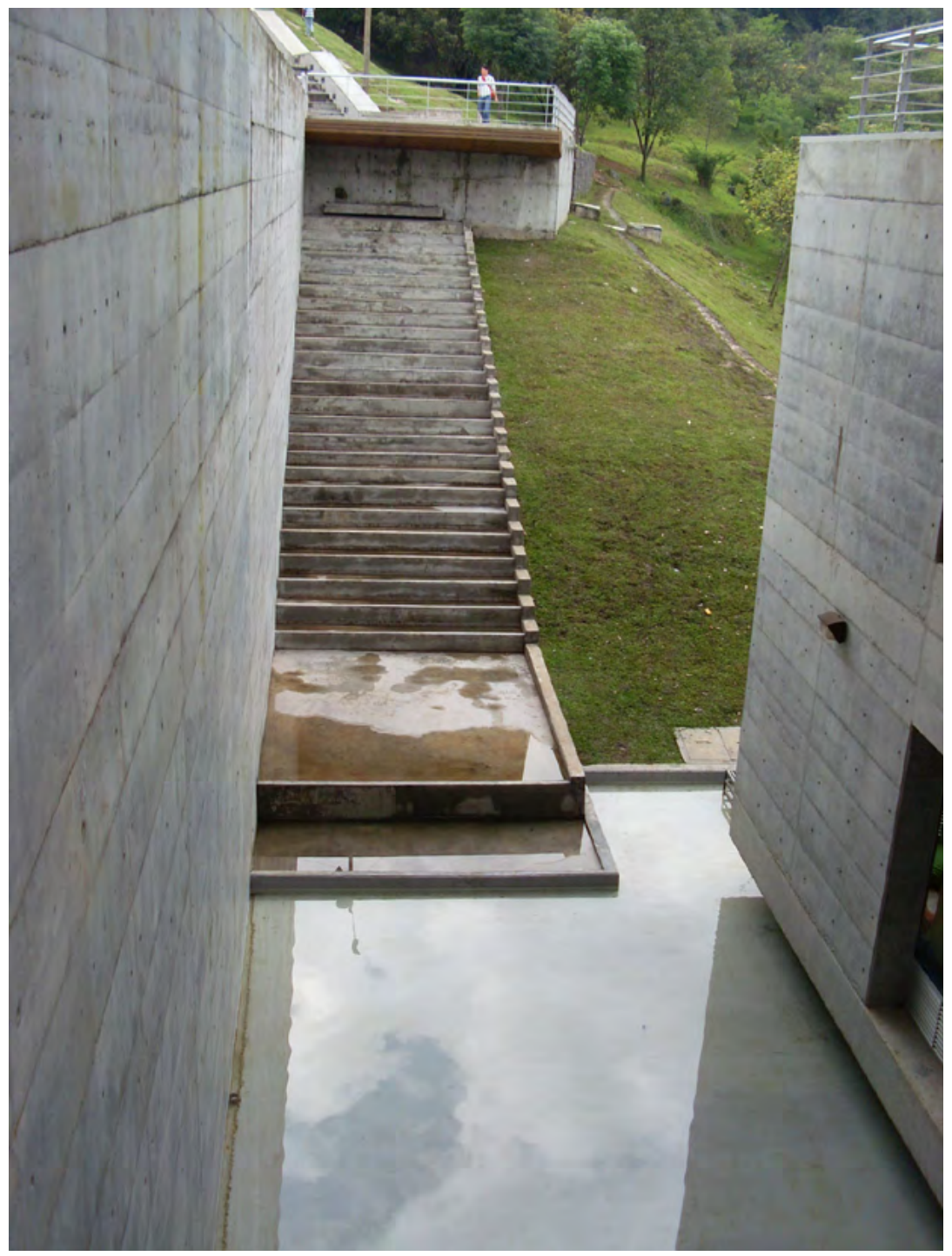

Figura 4. Parque Biblioteca la Quintana, Medellín 2009. La luz, los reflejos en el agua, la tonalidad de la materialidad, el cielo y el verde de la yerba generan una atmosfera especial en este conjunto, le escalera que pareciera no condujera a ningún sitio si no que solo conduce directo al espejo de agua como si en realidad se quisiera admirar algo que no se puede imaginar.

Fuente: Fotografia tomada por Mauricio Cabas 


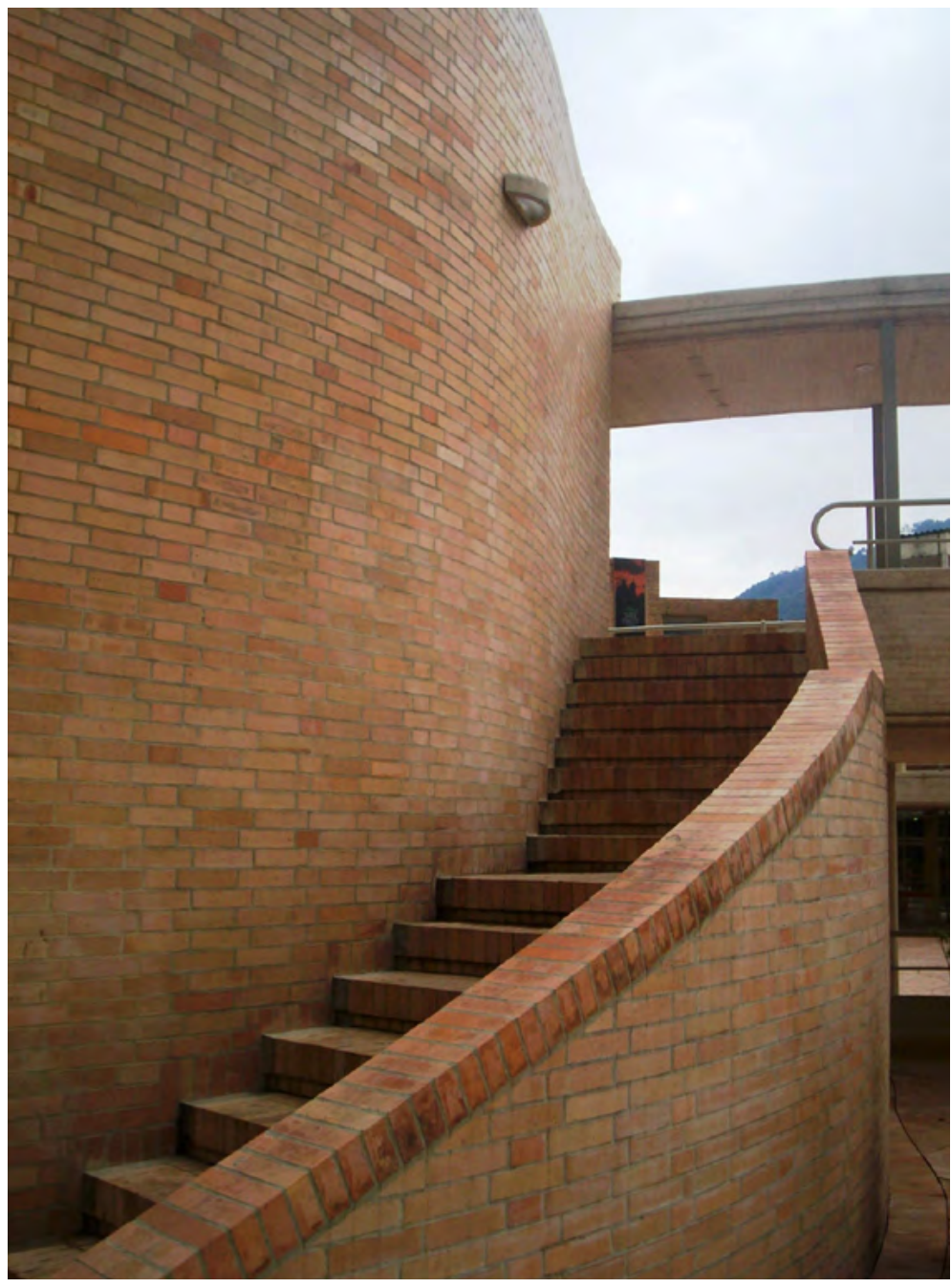

Figura 5. Centro Cultural Gabriel García Márquez, Bogotá 2008.

La materialidad y la calidez del ladrillo como elemento constructivo articulado con formas curvas que interactúan con el concreto de color casi ocre y el telón de fondo del cielo generan una sensación de contradicción entre el frio y lo cálido.

Fuente: Fotografia tomada por Mauricio Cabas

Todo lo expresado anteriormente nos lleva a pensar que las experiencias de la obra arquitectónica pasan de ser una dualidad entre el "yo" y "eso" a una unidad vivencial de fundición entre sujeto y objeto. $Y$ aunque la fenomenología podría fundamentar la interpretación y comprensión de la arquitectura como una intención materializada interactuando activamente con nosotros mediante una relación significativa y experiencial, tal relación no es lo suficientemente fuerte para romper el dualismo. Solamente, si muy profundamente a nivel subconsciente nos entregamos por completo a la realidad de una obra, dejándonos atrapar sin referenciar o depender de nada es que la experiencia de la unión con espacio puede ocurrir como lo señala Bermúdez. Vemos entonces el poder del arquitecto y la arquitectura, creando condiciones que estimulen al habitante a transformar su experiencia en tercera persona a una experiencia intima en primera persona, de unidad espiritual con el espacio (Bermúdez, 2014).

Entonces las experiencias arquitectónicas intangibles, en sí mismas, demandan un distanciamiento intelectual, casi como un alejamiento de lo racional, apartándose de las maneras más comunes de apre- 
ciar la arquitectura, basadas en el conocimiento, la lógica, el análisis, observación y evaluación estética de las obras, (Bermudez, 2013) y de ese modo entonces obtener lo que el espacio como tal está comunicando. Es muy factible que una persona que muestre desinterés por la arquitectura o no tenga conocimientos de la misma pueda obtener esas experiencias intangibles, ya que experimenta el espacio sin ninguna norma prestablecida. Y siendo arquitecto deberá poder abstraerse de todo lo que conoce y dejarse llevar por la belleza, por una arquitectura que no sobre estimule, una arquitectura en la cual la luz te acaricie y abrace muy suavemente. Estos conceptos pueden ser muy subjetivos y extremadamente personales, basados en sensibilidades intimas y que llevan a respuestas de comportamientos determinados. En la experiencia del espacio arquitectónico no solo están presentes muros y cubiertas ,sino también los efectos de luz, sonidos, vivencias, fenómenos que muchas veces solo se descubren muchos años después de ser materializado el mismo, pero que solo estaban posiblemente en la cabeza del arquitecto creador y en su intención (Cabas, 2011) La realidad arquitectónica solo puede tratarse de que un edificio logre conmover o no (Zumthor, 2006).

\section{Referencias}

Alvarez, L. (2013). Arquitectura y fenomenología. Sobre La arquitectónica de la «indeterminación» en el espacio. Eikasia, 815-836.

Ando, T. (1995). The Pritzker Prize. Loa Angeles: The Hyatt Foundation. Jenson \& Walker.

Argan, G. C. (1984). El concepto del espacio arquitectonico, desde el barroco a nuestros dias. Buenos Aires: Ediciones Nueva Vision.

Barreneche, G. (1996). Luz y Espacio en arquitectura. Manizales: Universidad Nacional sede Manizales.

Bermudez, J. (2008). Definiendo lo extraordinario en la arquitectura. Estudios estadisticos de la fenomenologia de lo bello. Revista Latinoamericana de estudios avanzados 14(28), 17-38.

Bermudez, J. (2013). El Rol del 'Distanciamiento' en lo Inefable Arquitectónico. Modulo Arquitectura CUC (12), 1(12), 11-25.

Bermudez, J. (2014). Arquitectura extraordinaria: donde materialidad y espritualidad se encuentran. Modulo Arquitectura CUC, 1(13), 101-113. 
Cabas, M. (2010). Conceptualizacion del espacio arquitectonico a traves de la historia. Modulo Arquitectura CUC (9), 1(9), 87-103.

Cabas, M. (2011). Lo intangible del espacio arquitectonico. Hito. Revista de arquitectura (25), 1(25), 7-12.

Gadamer, H. (1968). The Relevance of the Beautifil and Other Essays. New York: Cambrigde University Press

Garzón, R. (2012). La arquitectura de Frank Gehry:Espacialidad, envoltorio y yuxtaposición radical. Modulo Arquitectura CUC (11), 171-181.

Giedion, S. (1969). La arquitectura, fenomeno de transicion (las tres edades del espacio en arquitectura). Barcelona: Gustavo Gili.

Goycoolea, R. (1998). Filosofia y arquitectura. A parte rei: revista de filosofia.

Grillo, A. (2005). La arquitectura y la naturaleza compleja: arquitectura, ciencia y mímesis a finales del siglo $\mathrm{xx}$. Tesis de doctorado. Barcelona, catalunya, españa: universitat politècnica de catalunya.
Hardoy, J. (1968). Urban Plannig in PreColombian America. New York: Braziller.

LE Corbusier. (1993). El viaje a oriente. Valencia: Artes Garficas Soler S.A.

LE Corbusier. (2000). The Modulor. Boston: Springer Science \& Business Media.

Moline, M. (2011). Bitacora Almendron. Obtenido de Claves de la arquitectura: http://www.almendron. com/artehistoria/arte/arquitectura/ las-claves-de-la-arquitectura/ .

Munizaga, G. (1997). Las ciudades y su historia(una aproximacion). Santiago: Universidad Catolica de Chile.

Rasmussen, S. E. (1980). Experiencing Architecture. Cambrigde: MIT Press.

Zevi, B. (1958). Saber ver la arquitectura. Buenos Aires: Editorial Poseidón.

Zevi, B. (1999). Leer, escribir, hablar arquitectura. Barcelona: Ediciones Apostrofe.

Zumthor, P. (2006). Atmosferas. Entornos arquitectonicos. Las cosas a mi alrededor. Barcelona: Gustavo Gill. 